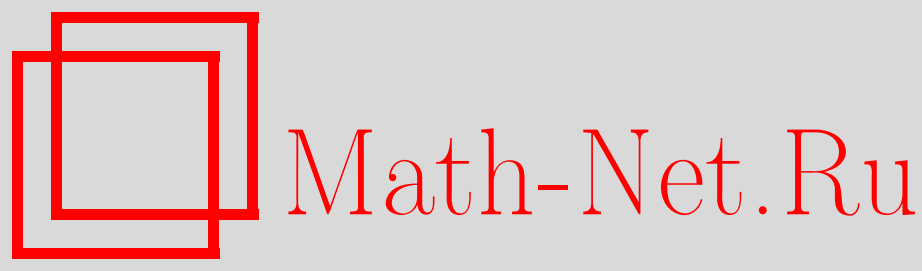

Ю. А. Чиркунов, С. Ю. Доброхотов, С. Б. Медведев, Д. С. Миненков, Точные решения одномерных уравнений мелкой воды над ровным и наклонным дном, $Т M \Phi$, 2014, том 178, номер 3, 322-345

DOI: https://doi.org/10.4213/tmf8607

Использование Общероссийского математического портала Math-Net.Ru подразумевает, что вы прочитали и согласны с пользовательским соглашением http://www.mathnet.ru/rus/agreement

Параметры загрузки:

IP : 54.81 .137 .203

26 апреля 2023 г., 15:11:01

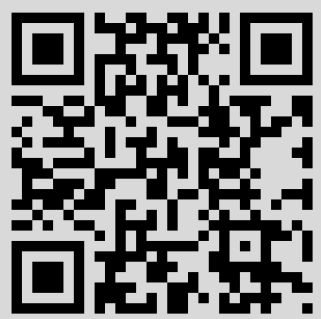




\title{
ФИЗИКА
}

Том 178, № 3

март, 2014

(C) 2014 г. Ю. А. Чиркунов ${ }^{* \dagger}$, С. Ю. Доброхотов ${ }^{\ddagger \S}$, С. Б. Медведев ${ }^{\dagger}$, Д. С. Миненков ${ }^{\ddagger \S}$

\section{ТОЧНЫЕ РЕШЕНИЯ ОДНОМЕРНЫХ НЕЛИНЕЙНЫХ УРАВНЕНИЙ МЕЛКОЙ ВОДЫ НАД РОВНЫМ И НАКЛОННЫМ ДНОМ}

\begin{abstract}
Установлена эквивалентность систем уравнений одномерных моделей мелкой воды, описывающих распространение поверхностных волн над ровным и наклонным дном. Для каждой из этих систем получены формулы общего вида их невырожденных решений, которые выражаются через решения уравнения Дарбу. Найденные инвариантные решения уравнения Дарбу являются простейшими представителями существенно различных (не связанных обратимыми точечными преобразованиями) его точных решений. Они зависят от 21 произвольной вещественной постоянной и после применения формул "размножения", полученных методами группового анализа, порождают 27-параметрическое семейство его существенно различных точных решений. Последующее использование выведенных инфинитезимальных формул "размножения" решений из этого семейства порождает счетное множество точных решений, а его линейная оболочка образует бесконечномерное векторное пространство решений уравнения Дарбу. В свою очередь, это векторное пространство решений уравнения Дарбу и общие формулы для невырожденных решений систем уравнений мелкой воды с ровным и наклонным дном дают бесконечное множество их решений. Далее формулы "размножения" для этих систем определяют их дополнительные невырожденные решения. Наконец, найдены все вырожденные решения этих систем. Тем самым создана база данных бесконечного множества точных решений систем уравнений одномерной нелинейной модели мелкой воды с ровным и наклонным дном.
\end{abstract}

Ключевые слова: уравнения мелкой воды, уравнение Эйлера-Пуассона-Дарбу, точные решения, инвариантные решения, группы симметрий.

DOI: $10.4231 / \operatorname{tmf} 8607$

*Новосибирский государственный технический университет, Новосибирск, Россия. E-mail: chr101@mail.ru

${ }^{\dagger}$ Институт вычислительных технологий СО РАН, Новосибирск, Россия.

E-mail: medvedev@ict.nsc.ru

${ }^{\ddagger}$ Институт проблем механики им. А. Ю. Ишлинского РАН, Москва, Россия.

E-mail: dobr@ipmnet.ru, minenkov.ds@gmail.com

$\S$ Московский физико-технический институт, Долгопрудный, Московская обл., Россия

ף Московский государственный университет им. М. В. Ломоносова, Москва, Россия 


\section{1. ВВЕДЕНИЕ}

Одномерные модели мелкой воды применяются при исследовании распространения жидкости в каналах, а также при исследовании цунами. Изучению этих моделей посвящено большое количество работ (см. работы [1]-[8] и указанную в них литературу). В настоящей работе рассматриваются две такие модели.

Первая модель задается системой дифференциальных уравнений

$$
u_{t}+u u_{x}+h_{x}=0, \quad h_{t}+u h_{x}+h u_{x}=0
$$

и описывает распространение со скоростью $u=u(t, x)$ поверхностных волн со свободной поверхностью $z=h(t, x)$ вдоль оси $о x$ над ровным дном $z=0$, где $о x z-$ прямоугольная декартова система координат, $t$ - время.

Вторая модель задается системой дифференциальных уравнений

$$
v_{\tau}+v v_{y}+\eta_{y}=0, \quad \eta_{\tau}+((y+\eta) v)_{y}=0
$$

и описывает распространение со скоростью $v=v(\tau, y)$ поверхностных волн со свободной поверхностью $z=y+\eta(\tau, y)$ вдоль оси оу над наклонным дном $z=y$, где $\eta(\tau, y)$ - превышение свободной поверхности над дном, оуz - прямоугольная декартова система координат, $\tau$ - время.

Непосредственными вычислениями устанавливается, что системы (1) и (2) связаны обратимым точечным преобразованием

$$
\tau=t, \quad y=x+\frac{t^{2}}{2}, \quad v=u+t, \quad \eta=h-x-\frac{t^{2}}{2} .
$$

Отсюда следует, что если $u=u(t, x), h=h(t, x)$ - решение системы (1), то

$$
v=u\left(\tau, y-\frac{\tau^{2}}{2}\right)+\tau, \quad \eta=h\left(\tau, y-\frac{\tau^{2}}{2}\right)-y-\tau^{2}
$$

- решение системы (2). Обратно, если $v=v(\tau, y), \eta=\eta(\tau, y)$ - решение системы (2), то

$$
u=v\left(t, x+\frac{t^{2}}{2}\right)-t, \quad h=\eta\left(t, x+\frac{t^{2}}{2}\right)+x+\frac{t^{2}}{2}
$$

- решение системы (1).

Следовательно, системы (1) и (2) в силу преобразования (3) эквивалентны. Все решения системы (2) получаются из решений системы (1) в результате преобразования (4), а все решения системы (1) получаются из решений системы (2) в результате преобразования (5).

\section{2. ГРУППОВЫЕ СВОЙСТВА}

Исследуем групповые свойства системы (1). Сначала найдем оператор (см. [9], $[10])$, допускаемый этой системой. Этот оператор ищем в виде

$$
\xi^{0}(t, x, u, h) \partial_{t}+\xi^{1}(t, x, u, h) \partial_{x}+\sigma^{1}(t, x, u, h) \partial_{u}+\sigma^{2}(t, x, u, h) \partial_{h} .
$$


Из условия инвариантности относительно этого оператора многообразия, определяемого уравнениями (1), после расщепления по параметрическим производным следует система определяющих уравнений

$$
\begin{gathered}
\xi_{h}^{1}+\xi_{u}^{0}=u \xi_{h}^{0}, \quad \xi_{u}^{1}=u \xi_{u}^{0}-h \xi_{h}^{0}, \quad \sigma_{u}^{2}=h \sigma_{h}^{1}, \quad \sigma_{h}^{2}-\sigma_{u}^{1}=\xi_{x}^{1}-\xi_{t}^{0}, \\
\sigma^{1}=\xi_{t}^{1}-u\left(\xi_{x}^{1}-\xi_{t}^{0}-u \xi_{x}^{0}\right)+h \xi_{x}^{0}, \quad \sigma^{2}=2 h\left(\xi_{x}^{1}-\xi_{t}^{0}-u \xi_{x}^{0}\right), \\
\sigma_{t}^{1}+u \sigma_{x}^{1}+\sigma_{x}^{2}=0, \quad \sigma_{t}^{2}+u \sigma_{x}^{2}+h \sigma_{x}^{1}=0 .
\end{gathered}
$$

После второго продолжения эта система приводится в инволюцию и интегрируется. Ее общее решение имеет вид

$$
\begin{gathered}
\xi^{0}=\left(c^{1}+c^{3}\right) t+f(u, h), \quad \xi^{1}=c^{1} x+c^{2} t+g(u, h)+u f(u, h), \\
\sigma^{1}=c^{2}-c^{3} u, \quad \sigma^{2}=-2 c^{3} h
\end{gathered}
$$

где $f=f(u, h)$ и $g=g(u, h)$ - любое решение системы линейных уравнений

$$
f_{u}=-g_{h}, \quad g_{u}=-(h f)_{h} .
$$

Таким образом, система (1) допускает псевдогруппу Ли преобразований, порождаемую операторами

$$
\begin{gathered}
X_{1}=t \partial_{t}+x \partial_{x}, \quad X_{2}=t \partial_{x}+\partial_{u}, \quad X_{3}=t \partial_{t}-u \partial_{u}-2 h \partial_{h}, \\
Z=f(u, h) \partial_{t}+(g(u, h)+u f(u, h)) \partial_{x} .
\end{gathered}
$$

В силу теоремы Ли [9] это означает, что система (1) допускает следующие преобразования, порождаемые операторами (6). Для оператора $X_{1}$ такими преобразованиями является группа преобразований равномерного растяжения по независимым переменным

$$
t^{\prime}=a^{1} t, \quad x^{\prime}=a^{1} x, \quad u^{\prime}=u, \quad h^{\prime}=h,
$$

для оператора $X_{2}$ - группа преобразований галилеева переноса

$$
t^{\prime}=t, \quad x^{\prime}=x+a^{2} t, \quad u^{\prime}=u+a^{2}, \quad h^{\prime}=h,
$$

для оператора $X_{3}$ - группа преобразований растяжения по независимым и зависимым переменным

$$
t^{\prime}=a^{3} t, \quad x^{\prime}=x, \quad u^{\prime}=\frac{u}{a^{3}}, \quad h^{\prime}=\frac{h}{\left(a^{3}\right)^{2}},
$$

а для оператора $Z$ - преобразования псевдогруппы, определяемые по формулам

$$
t^{\prime}=t+\phi(u, h), \quad x^{\prime}=x+\psi(u, h)+u \phi(u, h), \quad u^{\prime}=u, \quad h^{\prime}=h,
$$

где $\phi=\phi(u, h)$ и $\psi=\psi(u, h)$ - любое решение системы линейных уравнений

$$
\phi_{u}=-\psi_{h}, \quad \psi_{u}=-(h \phi)_{h} .
$$

Среди преобразований (10), в частности, содержатся группа преобразований переноса по $t$ (при $\left.\phi=a^{4}, \psi=-a^{4} u\right)$

$$
t^{\prime}=t+a^{4}, \quad x^{\prime}=x, \quad u^{\prime}=u, \quad h^{\prime}=h
$$


и группа преобразований переноса по $x$ (при $\left.\phi=0, \psi=a^{5}\right)$

$$
t^{\prime}=t, \quad x^{\prime}=x+a^{5}, \quad u^{\prime}=u, \quad h^{\prime}=h .
$$

Входящие в формулы (7)-(9), (11), (12) групповые параметры $a^{1}, a^{2}, a^{3}, a^{4}, a^{5}$ $\left(a^{1} a^{3} \neq 0\right)$ суть произвольные постоянные.

Если $u=u(t, x), h=h(t, x)$ - решение системы (1), то из формул (7)-(13) следует, что решением системы (1), записанной для переменных $t^{\prime}, x^{\prime}, u^{\prime}, h^{\prime}$, являются следующие пары функций:

$$
\begin{gathered}
u^{\prime}=u\left(\frac{t^{\prime}}{a^{1}}, \frac{x^{\prime}}{a^{1}}\right), \quad h^{\prime}=h\left(\frac{t^{\prime}}{a^{1}}, \frac{x^{\prime}}{a^{1}}\right) ; \\
u^{\prime}=u\left(t^{\prime}, x^{\prime}-a^{2} t^{\prime}\right)+a^{2}, \quad h^{\prime}=h\left(t^{\prime}, x^{\prime}-a^{2} t^{\prime}\right) ; \\
u^{\prime}=\frac{u\left(t^{\prime} / a^{3}, x^{\prime}\right)}{a^{3}}, \quad h^{\prime}=\frac{h\left(t^{\prime} / a^{3}, x^{\prime}\right)}{\left(a^{3}\right)^{2}} ; \\
u^{\prime}=u\left(t^{\prime}-\phi\left(u^{\prime}, h^{\prime}\right), x^{\prime}-\psi\left(u^{\prime}, h^{\prime}\right)-u \phi\left(u^{\prime}, h^{\prime}\right)\right), \\
h^{\prime}=h\left(t^{\prime}-\phi\left(u^{\prime}, h^{\prime}\right), x^{\prime}-\psi\left(u^{\prime}, h^{\prime}\right)-u \phi\left(u^{\prime}, h^{\prime}\right)\right) ; \\
u^{\prime}=u\left(t^{\prime}-a^{4}, x^{\prime}\right), \quad h^{\prime}=h\left(t^{\prime}-a^{4}, x^{\prime}\right) ; \\
u^{\prime}=u\left(t^{\prime}, x^{\prime}-a^{5}\right), \quad h^{\prime}=h\left(t^{\prime}, x^{\prime}-a^{5}\right) .
\end{gathered}
$$

Здесь $a^{1}, a^{2}, a^{3}, a^{4}, a^{5}\left(a^{1} a^{3} \neq 0\right)$ - произвольные вещественные постоянные, а $\phi=\phi(u, h), \psi=\psi(u, h)$ - любое решение системы (11). При этом формулы (14)-(16), $(18),(19)$ дают явные выражения для решений $u^{\prime}=u^{\prime}\left(t^{\prime}, x^{\prime}\right), h^{\prime}=h^{\prime}\left(t^{\prime}, x^{\prime}\right)$, а формула (17) задает эти решения неявно.

Применение преобразования (3) к формулам (7)-(9), (12), (13) приводит к следующим формулам производства ("размножения") решений системы (2). А именно, если $v=v(\tau, y), \eta=\eta(\tau, y)$ - решение системы (2), то решением системы $(2)$, записанной для переменных $\tau^{\prime}, y^{\prime}, v^{\prime}, \eta^{\prime}$, являются следующие пары функций $v^{\prime}=v^{\prime}\left(\tau^{\prime}, y^{\prime}\right)$, $\eta^{\prime}=\eta^{\prime}\left(\tau^{\prime}, y^{\prime}\right)$

$$
\begin{gathered}
v^{\prime}=v\left(\frac{\tau^{\prime}}{a^{1}}, \frac{2 a^{1} y^{\prime}+\left(1-a^{1}\right)\left(\tau^{\prime}\right)^{2}}{2\left(a^{1}\right)^{2}}\right)-\frac{\left(1-a^{1}\right) \tau^{\prime}}{a^{1}}, \\
\eta^{\prime}=\eta\left(\frac{\tau^{\prime}}{a^{1}}, \frac{2 a^{1} y^{\prime}+\left(1-a^{1}\right)\left(\tau^{\prime}\right)^{2}}{2\left(a^{1}\right)^{2}}\right)+\frac{\left(1-a^{1}\right)\left(2 a^{1} y^{\prime}+\left(\tau^{\prime}\right)^{2}\right)}{2\left(a^{1}\right)^{2}} \\
v^{\prime}=v\left(\tau^{\prime}, y^{\prime}-a^{2} \tau^{\prime}\right)+a^{2}, \quad \eta^{\prime}=\eta\left(\tau^{\prime}, y^{\prime}-a^{2} \tau^{\prime}\right)-a^{2} \tau^{\prime} ; \\
v^{\prime}=\frac{1}{a^{3}}\left(v\left(\frac{\tau^{\prime}}{a^{3}}, y^{\prime}+\frac{\left(\tau^{\prime}\right)^{2}}{2\left(a^{3}\right)^{2}}-\frac{\left(\tau^{\prime}\right)^{2}}{2}\right)-\frac{\tau^{\prime}}{a^{3}}\right)+\tau^{\prime}, \\
\eta^{\prime}=\frac{1}{\left(a^{3}\right)^{2}}\left(\eta\left(\frac{\tau^{\prime}}{a^{3}}, y^{\prime}+\frac{\left(\tau^{\prime}\right)^{2}}{2\left(a^{3}\right)^{2}}-\frac{\left(\tau^{\prime}\right)^{2}}{2}\right)+y^{\prime}+\frac{\left(\tau^{\prime}\right)^{2}}{2\left(a^{3}\right)^{2}}-\frac{\left(\tau^{\prime}\right)^{2}}{2}\right)-y^{\prime} ; \\
v^{\prime}=v\left(\tau^{\prime}-a^{4}, y^{\prime}-a^{4} \tau^{\prime}+\frac{\left(a^{4}\right)^{2}}{2}\right)+a^{4}, \\
\eta^{\prime}=\eta\left(\tau^{\prime}-a^{4}, y^{\prime}-a^{4} \tau^{\prime}+\frac{\left(a^{4}\right)^{2}}{2}\right)-a^{4} \tau^{\prime}+\frac{\left(a^{4}\right)^{2}}{2} ; \\
v^{\prime}=v\left(\tau^{\prime}, y^{\prime}-a^{5}\right), \quad \eta^{\prime}=\eta\left(\tau^{\prime}, y^{\prime}-a^{5}\right)-a^{5} .
\end{gathered}
$$


Если $v^{\prime}=v^{\prime}\left(\tau^{\prime}, y^{\prime}\right), \eta^{\prime}=\eta^{\prime}\left(\tau^{\prime}, y^{\prime}\right)$ - решение системы (2), записанной для переменных $\tau^{\prime}, y^{\prime}, v^{\prime}, \eta^{\prime}$, то применение к этому решению преобразования (3) в силу формул (10) дает следующие формулы производства ("размножения") решений системы (2):

$$
\begin{aligned}
v=\tau & -\phi(v-\tau, \eta+y)+v^{\prime}(\phi(v-\tau, \eta+y), \psi(v-\tau, \eta+y)+ \\
& \left.+(v-\tau) \phi(v-\tau, \eta+y)+\frac{\phi^{2}(v-\tau, \eta+y)}{2}\right) \\
\eta=\psi & (v-\tau, \eta+y)-y+(v-\tau) \phi(v-\tau, \eta+y)+\frac{\phi^{2}(v-\tau, \eta+y)}{2}+ \\
& +\eta^{\prime}(\phi(v-\tau, \eta+y), \psi(v-\tau, \eta+y)+ \\
& \left.+(v-\tau) \phi(v-\tau, \eta+y)+\frac{\phi^{2}(v-\tau, \eta+y)}{2}\right)
\end{aligned}
$$

которые задают неявно пару функций $v=v(\tau, y), \eta=\eta(\tau, y)$ - решение системы (2). Здесь $\phi=\phi(u, h), \psi=\psi(u, h)$ - любое решение системы (11).

\section{3. ОБЩИЙ ВИД НЕВЫРОЖДЕННЫХ РЕШЕНИЙ}

Решение $u=u(t, x), h=h(t, x)$ системы (1) называется невырожденным, если отличен от нуля якобиан $\partial(u, h) / \partial(t, x) \equiv u_{t} h_{x}-h_{t} u_{x} \neq 0$. Аналогично решение $v=v(\tau, y), \eta=\eta(\tau, y)$ системы (2) называется невырожденным, если отличен от нуля якобиан $\partial(v, \eta) / \partial(\tau, y) \neq 0$. Множество невырожденных решений системы $(2)$ получается из множества невырожденных решений системы (1) в результате обратимого преобразования (3).

Непосредственными вычислениями устанавливается, что всякое невырожденное решение $u=u(t, x), h=h(t, x)$ системы (1) по формулам

$$
t=\frac{2}{\rho} w_{\rho}(u, \rho), \quad x=\frac{2 u}{\rho} w_{\rho}(u, \rho)-w_{u}(u, \rho), \quad \rho=2 \sqrt{h}
$$

определяет (с точностью до аддитивной константы) решение $w(u, \rho)$ уравнения Дарбу

$$
w_{u u}=w_{\rho \rho}+\frac{1}{\rho} w_{\rho} .
$$

Обратно, если для решения $w(u, \rho)$ уравнения Дарбу в некоторой области $(u, \rho)$ отличен от нуля якобиан

$$
\frac{\partial(t, x)}{\partial(u, h)} \equiv \frac{4}{\rho^{2}} w_{\rho \rho}^{2}-\frac{4}{\rho^{2}} w_{u \rho}^{2}-\frac{8}{\rho^{3}} w_{\rho \rho} w_{\rho}+\frac{4}{\rho^{4}} w_{\rho}^{2} \neq 0,
$$

то формулы (27) неявным образом определяют решение $u(t, x)$ и $h(r, x)$ в соответствующей области $(t, x)$.

Применение преобразования (3) к общему невырожденному решению $u(t, x), h(t, x)$ системы (1) дает общее невырожденное решение $v=v(\tau, y), \eta=\eta(\tau, y)$ системы (2), которое по формулам

$$
\tau=\left.\frac{1}{\sqrt{y+\eta}} \partial_{\rho} w(v-\tau, \rho)\right|_{\rho=2 \sqrt{y+\eta}}, \quad y+\frac{\tau^{2}}{2}=\tau v-\left.\partial_{u} w(u, 2 \sqrt{y+\eta})\right|_{u=v-\tau}
$$


определяет (с точностью до аддитивной константы) решение $w(u, \rho)$ уравнения Дарбу (28). Якобиан обратного преобразования, определяющего неявным образом решение $v(\tau, y), \eta(\tau, y)$ по заданному решению $w(u, \rho)$ уравнения Дарбу, равен $\partial(\tau, y) / \partial(v, \eta)=\partial(t, x) / \partial(u, h)$.

\section{4. ТОЧНЫЕ РЕШЕНИЯ}

Из формул (27) и (29) следует, что поиск невырожденных решений систем (1) и (2) сводится к решению уравнения Дарбу (28), симметрии которого исследовались в многочисленных работах (см., в частности, [11]-[14]). Как показано в работах [9], [15]-[19], уравнение Дарбу (28) эквивалентно по функции линейному дифференциальному уравнению второго порядка, имеющему ненулевой обобщенный инвариант Лапласа и допускающему группу Ли преобразований максимального порядка, операторы алгебры Ли которой являются линейно автономными [20]. Уравнение (28) ввиду линейности допускает бесконечную группу Ли преобразований с нормальным делителем, порождаемым операторами [9], [11]-[19]

$$
Y=w^{0}(u, \rho) \partial_{w}
$$

где $w=w^{0}(u, \rho)$ - произвольное решение уравнения (28), факторгруппа по которому является четырехпараметрической и порождается операторами

$$
Y_{1}=\partial_{u}, \quad Y_{2}=u \partial_{u}+\rho \partial_{\rho}, \quad Y_{3}=\left(u^{2}+\rho^{2}\right) \partial_{u}+2 u \rho \partial_{\rho}-u w \partial_{w}, \quad Y_{4}=w \partial_{w}
$$

В силу теоремы Ли [9] это означает, что уравнение (28) допускает следующие преобразования, порождаемые допускаемыми операторами. Для оператора $Y$ такими преобразованиями являются преобразования, обусловленные линейностью уравнения

$$
u^{\prime}=u, \quad \rho^{\prime}=\rho, \quad w^{\prime}=w+w^{0}(u, \rho),
$$

где $w=w^{0}(u, \rho)$ - любое решение уравнения (28), для оператора $Y_{1}$ - группа преобразований переноса по $u$

$$
u^{\prime}=u+b^{1}, \quad \rho^{\prime}=\rho, \quad w^{\prime}=w,
$$

для оператора $Y_{2}$ - группа преобразований равномерного растяжения по независимым переменным

$$
u^{\prime}=b^{2} u, \quad \rho^{\prime}=b^{2} \rho, \quad w^{\prime}=w,
$$

для оператора $Y_{3}$ - группа преобразований

$$
\begin{gathered}
u^{\prime}=\frac{\left(u+b^{3}\left(u^{2}-\rho^{2}\right)\right)\left(u^{2}-\rho^{2}\right)}{\left(u+b^{3}\left(u^{2}-\rho^{2}\right)\right)^{2}-u^{2}}, \quad \rho^{\prime}=\frac{\rho\left(u^{2}-\rho^{2}\right)}{\left(u+b^{3}\left(u^{2}-\rho^{2}\right)\right)^{2}-u^{2}} \rho, \\
w^{\prime}=w \sqrt{\frac{u^{2}-\rho^{2}}{\left(u+b^{3}\left(u^{2}-\rho^{2}\right)\right)^{2}-u^{2}}},
\end{gathered}
$$

а для оператора $Y_{4}$ - группа обусловленных однородностью уравнения преобразований растяжения по функции

$$
u^{\prime}=u, \quad \rho^{\prime}=\rho, \quad w^{\prime}=b^{4} w .
$$


Входящие в формулы (31)-(34) групповые параметры $b^{1}, b^{2}, b^{3}, b^{4}\left(b^{2} b^{4} \neq 0\right)$ суть произвольные вещественные постоянные.

Если $w(u, \rho)$ - решение уравнения (28), то из формул (31)-(33) следует, что решениями уравнения $(24)$, записанного для переменных $u^{\prime}, \rho^{\prime}, w^{\prime}$, являются следующие функции $w^{\prime}=w^{\prime}\left(u^{\prime}, \rho^{\prime}\right)$ :

$$
\begin{gathered}
w^{\prime}=w\left(u^{\prime}-b^{1}, \rho^{\prime}\right) ; \quad w^{\prime}=w\left(\frac{u^{\prime}}{b^{2}}, \frac{\rho^{\prime}}{b^{2}}\right) ; \\
w^{\prime}=\rho^{\prime} \sqrt{f\left(u^{\prime}, \rho^{\prime}\right)} w\left(\rho^{\prime} f\left(u^{\prime}, \rho^{\prime}\right)\left(u^{\prime} \rho^{\prime}-b^{3}\left(\left(u^{\prime}\right)^{2}-\left(\rho^{\prime}\right)^{2}\right)\right),\left(\rho^{\prime}\right)^{3} f\left(u^{\prime}, \rho^{\prime}\right)\right), \\
f\left(u^{\prime}, \rho^{\prime}\right)=\frac{\left(u^{\prime}\right)^{2}-\left(\rho^{\prime}\right)^{2}}{\left(u^{\prime} \rho^{\prime}-b^{3}\left(\left(u^{\prime}\right)^{2}-\left(\rho^{\prime}\right)^{2}\right)\right)^{2}-\left(\rho^{\prime}\right)^{4}} .
\end{gathered}
$$

Ввиду линейности уравнения $(28)$ для любого его решения $w(u, \rho)$, являющегося комплекснозначной функцией, решениями этого уравнения являются также и функции $\operatorname{Re} w(u, \rho), \operatorname{Im} w(u, \rho)$. Поэтому формулы (35) производства ("размножения") решений уравнения (28) обобщаются следующим образом: для любого решения $w(u, \rho)$ уравнения (28) и для любых вещественных постоянных $\beta^{k}, \gamma^{k}(k=1,2,3)$, $\left(\beta^{2}\right)^{2}+\left(\gamma^{2}\right)^{2} \neq 0$, решениями уравнения $(28)$, записанного для переменных $u^{\prime}, \rho^{\prime}, w^{\prime}$, являются следующие функции $w^{\prime}=w^{\prime}\left(u^{\prime}, \rho^{\prime}\right)$ :

$$
\begin{aligned}
& w^{\prime}=\operatorname{Re} w\left(u^{\prime}-\left(\beta^{1}+i \gamma^{1}\right), \rho^{\prime}\right) ; \\
& w^{\prime}=\operatorname{Im} w\left(u^{\prime}-\left(\beta^{1}+i \gamma^{1}\right), \rho^{\prime}\right) ; \\
& w^{\prime}=\operatorname{Re} w\left(\frac{u^{\prime}}{\beta^{2}+i \gamma^{2}}, \frac{\rho^{\prime}}{\beta^{2}+i \gamma^{2}}\right) ; \\
& w^{\prime}=\operatorname{Im} w\left(\frac{u^{\prime}}{\beta^{2}+i \gamma^{2}}, \frac{\rho^{\prime}}{\beta^{2}+i \gamma^{2}}\right) ; \\
& w^{\prime}=\operatorname{Re}\left(\rho^{\prime} \sqrt{f\left(u^{\prime}, \rho^{\prime}\right)} w\left(\rho^{\prime} f\left(u^{\prime}, \rho^{\prime}\right)\left(u^{\prime} \rho^{\prime}-b^{3}\left(\left(u^{\prime}\right)^{2}-\left(\rho^{\prime}\right)^{2}\right)\right),\left(\rho^{\prime}\right)^{3} f\left(u^{\prime}, \rho^{\prime}\right)\right)\right) ; \\
& w^{\prime}=\operatorname{Im}\left(\rho^{\prime} \sqrt{f\left(u^{\prime}, \rho^{\prime}\right)} w\left(\rho^{\prime} f\left(u^{\prime}, \rho^{\prime}\right)\left(u^{\prime} \rho^{\prime}-b^{3}\left(\left(u^{\prime}\right)^{2}-\left(\rho^{\prime}\right)^{2}\right)\right),\left(\rho^{\prime}\right)^{3} f\left(u^{\prime}, \rho^{\prime}\right)\right)\right) ;
\end{aligned}
$$

где

$$
f\left(u^{\prime}, \rho^{\prime}\right)=\frac{\left(u^{\prime}\right)^{2}-\left(\rho^{\prime}\right)^{2}}{\left(u^{\prime} \rho^{\prime}-b^{3}\left(\left(u^{\prime}\right)^{2}-\left(\rho^{\prime}\right)^{2}\right)\right)^{2}-\left(\rho^{\prime}\right)^{4}}, \quad b^{3}=\beta^{3}+i \gamma^{3} \quad\left(i^{2}=-1\right) .
$$

Из (30) в силу известной [10] инфинитезимальной формулы производства решений следуют формулы производства ("размножения") решений уравнения (28). А именно, если $w=W(u, \rho)$ - произвольное гладкое решение уравнения (28), то решениями этого уравнения являются следующие функции:

$$
\begin{aligned}
& w=W_{u}(u, \rho) \\
& w=u W_{u}(u, \rho)+\rho W_{\rho}(u, \rho) ; \\
& w=\left(u^{2}+\rho^{2}\right) W_{u}(u, \rho)+2 u \rho W_{\rho}(u, \rho)+u W(u, \rho) .
\end{aligned}
$$


Формулы (37) приведены, в частности, в работе [21]. Ввиду линейности уравнения (28) любая линейная комбинация решений этого уравнения является его решением.

Для классификации инвариантных решений уравнения (28) строится оптимальная система неподобных подалгебр алгебры Ли с базисом (30). Каждой подалгебре из этой оптимальной системы подалгебр соответствует порождаемая ею подгруппа основной группы уравнения (28). Применение критерия инвариантности функции относительно группы Ли преобразований [9] позволяет получить универсальный инвариант каждой подгруппы из построенной оптимальной системы подгрупп в пространстве $R^{3}(u, \rho, w)$. Результаты представлены в табл. 1,2 , в которых $\alpha, \beta-$ произвольные постоянные.

ТАБлицА 1. Однопараметрические подгруппы $\theta_{1 . k}$

\begin{tabular}{|l|l|l|}
\hline$k$ & Базис подалгебры & Универсальный инвариант \\
\hline 1 & $Y_{2}+\alpha Y_{4}$ & $\frac{u}{\rho}, \rho^{-\alpha} w$ \\
\hline 2 & $Y_{1}$ & $\rho, w$ \\
\hline 3 & $Y_{1}+Y_{4}$ & $\rho, e^{-u} w$ \\
\hline 4 & $Y_{3}$ & $\frac{u^{2}-\rho^{2}}{\rho}, w \sqrt{\rho}$ \\
\hline 5 & $Y_{3}+Y_{4}$ & $\frac{u^{2}-\rho^{2}}{\rho}, \rho^{1 / 2} \exp \left(\frac{u}{u^{2}-\rho^{2}}\right) w$ \\
\hline 6 & $Y_{1}+Y_{3}+\beta Y_{4}$ & $\frac{u^{2}-\rho^{2}+1}{2 \rho}, \rho^{1 / 2} \exp \left(-\frac{\beta}{2} \arcsin \frac{u^{2}-\rho^{2}-1}{\sqrt{\left(u^{2}-\rho^{2}-1\right)^{2}+4 u^{2}}}\right) w$ \\
\hline 7 & $Y_{4}$ & $u, \rho$ \\
\hline
\end{tabular}

ТАБлицА 2. Двухпараметрические подгруппы $\theta_{2 . k}$

\begin{tabular}{|l|l|l|}
\hline$k$ & Базис подалгебры & Универсальный инвариант \\
\hline 1 & $Y_{2}+\alpha Y_{4}, Y_{3}$ & $\rho^{\alpha+1}\left(\rho^{2}-u^{2}\right)^{-(\alpha+1 / 2)} w$ \\
\hline 2 & $Y_{2}+\beta Y_{4}, Y_{1}$ & $\rho^{-\beta} w$ \\
\hline 3 & $Y_{1}, Y_{4}$ & $\rho$ \\
\hline 4 & $Y_{2}, Y_{4}$ & $\frac{u}{\rho}$ \\
\hline 5 & $Y_{3}, Y_{4}$ & $\frac{u^{2}-\rho^{2}}{\rho}$ \\
\hline 6 & $\pm Y_{1}+Y_{3}, Y_{4}$ & $\frac{u^{2}-\rho^{2} \pm 1}{\rho}$ \\
\hline
\end{tabular}

Найденные универсальные инварианты всех подгрупп в табл. 1, 2 позволяют получить простейших представителей всех существенно различных (не связанных точечными преобразованиями) инвариантных решений уравнения (28). Входящие во все последующие формулы величины $c^{k}, k=0,1,2, \ldots, 18$, суть произвольные вещественные постоянные. 
Для подгрупп $\theta_{1.7}, \theta_{2 . k}, k=3,4,5,6$, не выполнены необходимые условия [9] существования инвариантного решения. Неособые [9] инвариантные $H$-решения ранга 0 уравнения (28) суть решения, инвариантные относительно подгрупп $H \in\left\{\theta_{2 . k}\right\}$, $k=1,2$. Ниже речь идет только о ненулевых решениях.

Инвариантное $\theta_{2.1}$-решение имеет вид

$$
w=c^{0} \rho^{-(\alpha+1)}\left(\rho^{2}-u^{2}\right)^{(\alpha+1 / 2)} .
$$

Подстановка в уравнение (28) показывает, что такое решение существует только при $\alpha=-1$. В этом случае оно определяется по формуле

$$
w=\frac{c^{0}}{\sqrt{\rho^{2}-u^{2}}} .
$$

Инвариантное $\theta_{2.2}$-решение имеет вид $w=c \rho^{\beta}(c=$ const $\neq 0)$ и удовлетворяет уравнению (28) только при $\beta=0$.

Неособые [9] инвариантные $H$-решения ранга 1 уравнения (28) суть решения, инвариантные относительно подгрупп $H \in\left\{\theta_{1 . k}\right\}, k=1,2, \ldots, 7$.

Инвариантное $\theta_{1.1}$-решение имеет следующее представление:

$$
w=\rho^{\alpha} W(\xi), \quad \xi=\frac{u}{\rho} .
$$

Подстановка в уравнение (28) показывает, что функция $W(\xi)$ является решением уравнения

$$
\left(\xi^{2}-1\right) W^{\prime \prime}+(1-2 \alpha) \xi W^{\prime}+\alpha^{2} W=0,
$$

которое в результате замены

$$
W(\xi)=\theta(\eta), \quad 2 \eta=\xi+1
$$

приводится к гипергеометрическому уравнению Гаусса

$$
\eta(\eta-1) \theta^{\prime \prime}+\left((1-2 \alpha) \eta-\left(\frac{1}{2}-\alpha\right)\right) \theta^{\prime}+\alpha^{2} \theta=0,
$$

решение которого выражается через гипергеометрическую функцию $F(a, b, c ; \xi)$ [22], [23]. Соответствующее инвариантное $\theta_{1.1}$-решение определяется по формуле

$$
w(\xi)=c^{1} \rho^{\alpha} F\left(-\alpha,-\alpha ; \frac{1}{2}-\alpha ; \frac{\rho+u}{2 \rho}\right)+c^{2} \frac{(\rho+u)^{\alpha+1 / 2}}{\sqrt{\rho}} F\left(\frac{1}{2}, \frac{1}{2} ; \frac{3}{2}+\alpha ; \frac{\rho+u}{2 \rho}\right),
$$

если только $\alpha \neq m ; m+1 / 2(m=0, \pm 1, \pm 2, \ldots)$. При $\alpha=0,-1$ это решение выражается через элементарные функции:

при $\alpha=0$

$$
w=c^{3}+c^{4} \ln \frac{u+\sqrt{u^{2}-\rho^{2}}}{\rho},
$$

при $\alpha=-1$

$$
w=\frac{1}{\sqrt{u^{2}-\rho^{2}}}\left(c^{1}+c^{2} \ln \frac{u+\sqrt{u^{2}-\rho^{2}}}{\rho}\right) .
$$


При $\alpha=-1 / 2$ решение задается формулой

$$
w=\frac{1}{\sqrt{\rho}}\left(c^{1} P_{-1 / 2}\left(\frac{u}{\rho}\right)+c^{2} Q_{-1 / 2}\left(\frac{u}{\rho}\right)\right),
$$

где $P_{-1 / 2}(\xi)$ и $Q_{-1 / 2}(\xi)$ - функции Лежандра соответственно первого и второго рода [22], [23].

Инвариантное $\theta_{1.2}$-решение имеет вид

$$
w=c^{5}+c^{6} \ln \rho .
$$

Инвариантное $\theta_{1.3}$-решение $w=e^{u} W(\rho)$ выражается через цилиндрические функции [22], [23] с чисто мнимым аргументом - функцию Бесселя $J_{0}(i \rho)$ и функцию Неймана $Y_{0}(i \rho)$, а именно

$$
w=e^{u}\left(c^{7} J_{0}(i \rho)+c^{8} Y_{0}(i \rho)\right)
$$

Инвариантное $\theta_{1.4}$-решение имеет вид

$$
w=\frac{1}{\sqrt{\rho}} W(\xi), \quad \xi=\frac{u^{2}-\rho^{2}}{\rho} .
$$

Подстановка в уравнение (28) приводит к уравнению Эйлера

$$
\xi^{2} W^{\prime \prime}+2 \xi W^{\prime}+\frac{1}{4} W=0
$$

которое интегрируется, и искомое $\theta_{1.4}$-решение определяется по формуле

$$
w=\frac{1}{\sqrt{u^{2}-\rho^{2}}}\left(c^{9}+c^{10} \ln \frac{u^{2}-\rho^{2}}{\rho}\right) .
$$

Инвариантное $\theta_{1.5}$-решение представляется в виде

$$
w=\frac{1}{\sqrt{\rho}} \exp \left(\frac{u}{\rho^{2}-u^{2}}\right) W(\xi), \quad \xi=\frac{u^{2}-\rho^{2}}{\rho} .
$$

Подстановка в уравнение (28) приводит к уравнению

$$
4 \xi^{4} W^{\prime \prime}+8 \xi^{3} W^{\prime}+\left(\xi^{2}-4\right) W=0
$$

которое интегрируется с помощью цилиндрических функций Бесселя и Неймана чисто мнимого аргумента. В результате инвариантное $\theta_{1.5}$-решение определяется по формуле

$$
w=\frac{1}{\sqrt{u^{2}-\rho^{2}}} \exp \left(\frac{u}{\rho^{2}-u^{2}}\right)\left(c^{11} J_{0}\left(\frac{i \rho}{u^{2}-\rho^{2}}\right)+c^{12} Y_{0}\left(\frac{i \rho}{u^{2}-\rho^{2}}\right)\right) .
$$

Инвариантное $\theta_{1.6}$-решение имеет вид

$$
w=\rho^{-1 / 2} \exp \left(\frac{\beta}{2} \arcsin \frac{u^{2}-\rho^{2}-1}{\sqrt{\left(u^{2}-\rho^{2}-1\right)^{2}+4 u^{2}}}\right) W(\xi), \quad \xi=\frac{u^{2}-\rho^{2}+1}{2 \rho} .
$$


Подстановка в уравнение (28) дает для функции $W(\xi)$ уравнение

$$
4\left(\xi^{2}+1\right)^{2} W^{\prime \prime}+8 \xi\left(\xi^{2}+1\right) W^{\prime}+\left(\xi^{2}+1-\beta^{2}\right) W=0,
$$

которое с помощью комплексных зависимых и независимых переменных

$$
W(\xi)=(4 \eta(\eta-1))^{\beta i / 4} \theta(\eta), \quad \xi=-i(2 \eta-1) \quad\left(i^{2}=-1\right),
$$

приводится к гипергеометрическому уравнению Гаусса

$$
\eta(\eta-1) \theta^{\prime \prime}+(2+\beta i)\left(\eta-\frac{1}{2}\right) \theta^{\prime}-\left(\frac{\beta-i}{2}\right)^{2} \theta=0
$$

решение которого при $\beta \neq 0$ выражается через гипергеометрическую функцию [22], [23]. В силу линейности уравнения (28) инвариантные $\theta_{1.6}$-решения при $\beta \neq 0$ суть

$$
\begin{aligned}
w= & \operatorname{Re}\left(f ( u , \rho , \beta ) \left(\left(c^{13}+i c^{14}\right) F\left(\frac{1+\beta i}{2}, \frac{1+\beta i}{2} ; 1+\frac{\beta i}{2} ; \frac{2 \rho+i\left(u^{2}-\rho^{2}+1\right)}{4 \rho}\right)+\right.\right. \\
& \left.\left.+\left(c^{15}+i c^{16}\right)\left(\frac{2 \rho+i\left(u^{2}-\rho^{2}+1\right)}{4 \rho}\right)^{-\beta i / 2} F\left(\frac{1}{2}, \frac{1}{2} ; 1-\frac{\beta i}{2} ; \frac{2 \rho+i\left(u^{2}-\rho^{2}+1\right)}{4 \rho}\right)\right)\right), \\
w= & \operatorname{Im}\left(f ( u , \rho , \beta ) \left(\left(c^{13}+i c^{14}\right) F\left(\frac{1+\beta i}{2}, \frac{1+\beta i}{2} ; 1+\frac{\beta i}{2} ; \frac{2 \rho+i\left(u^{2}-\rho^{2}+1\right)}{4 \rho}\right)+\right.\right. \\
& \left.\left.+\left(c^{15}+i c^{16}\right)\left(\frac{2 \rho+i\left(u^{2}-\rho^{2}+1\right)}{4 \rho}\right)^{-\beta i / 2} F\left(\frac{1}{2}, \frac{1}{2} ; 1-\frac{\beta i}{2} ; \frac{2 \rho+i\left(u^{2}-\rho^{2}+1\right)}{4 \rho}\right)\right)\right),
\end{aligned}
$$

где

$f(u, \rho, \beta)=\rho^{(-1-\beta i) / 2}\left(\left(u^{2}-\rho^{2}+1\right)^{2}+4 \rho^{2}\right)^{\beta i / 4} \exp \left(\frac{\beta}{2} \arcsin \frac{u^{2}-\rho^{2}-1}{\sqrt{\left(u^{2}-\rho^{2}-1\right)^{2}+4 u^{2}}}\right)$.

При $\beta=0$ инвариантное $\theta_{1.6}$-решение задается формулой

$$
w=\frac{1}{\sqrt{\rho}}\left(c^{17} P_{-1 / 2}\left(\frac{i\left(u^{2}-\rho^{2}+1\right)}{2 \rho}\right)+c^{18} Q_{-1 / 2}\left(\frac{i\left(u^{2}-\rho^{2}+1\right)}{2 \rho}\right)\right),
$$

где $P_{-1 / 2}(\xi)$ и $Q_{-1 / 2}(\xi)$ - функции Лежандра соответственно первого и второго рода [22], [23].

Найденные инвариантные решения (38)-(48) уравнения Дарбу (28) являются простейшими представителями существенно различных (не связанных обратимыми точечными преобразованиями) точных решений этого уравнения. Они зависят от 21 произвольной вещественной постоянной $\alpha, \beta, c^{0}, c^{1}, c^{2}, \ldots, c^{18}$. Применение к этим решениям формул (36) производства ("размножения") решений уравнения (28), зависящих от шести произвольных вещественных постоянных $\beta^{k}, \gamma^{k}, k=1,2,3$, дает 27-параметрическое множество существенно различных точных решений этого уравнения. Применение к этим решениям инфинитезимальных формул (37) производства ("размножения") решений дает счетное множество точных решений. Линейная оболочка этого множества образует бесконечномерное векторное пространство $\Omega$ точных решений уравнения Дарбу. 
Формулы (27) общего вида невырожденных решений системы (1) уравнений мелкой воды, описывающей распространение поверхностных волн над ровным дном, порождают при $w$, пробегающих пространство $\Omega$, бесконечное множество точных решений этой системы. Применение к этим решениям формул (14)-(19) производства ("размножения") решений дает дополнительные невырожденные точные решения системы (1).

Формулы (29) общего вида невырожденных решений системы уравнений мелкой воды (2), описывающей распространение поверхностных волн над наклоным дном, порождают при $w$, пробегающих пространство $\Omega$, бесконечное множество точных решений этой системы. Применение к этим решениям формул (20)-(24) производства (“размножения") решений дает дополнительные невырожденные точные решения системы (2).

\section{5. ПРИМЕРЫ ПОСТРОЕНИЯ НЕВЫРОЖДЕННЫХ РЕШЕНИЙ}

На рис. 1-7 представлены примеры решений уравнения Дарбу $\theta_{2.1}, \theta_{1.1}, \theta_{1.3}, \theta_{1.4}$, $\theta_{1.5}$ и $\theta_{1.6}$ для некоторых значений констант и соответствующие им решения нелинейной системы (1). На рис. 8, 9 представлены решение уравнения Дарбу и решения нелинейной системы, соответствующие специальному решению

$$
U_{0}(\tau, y)=\operatorname{Re}\left(\frac{2 A}{\left(y-(\tau+2 a)^{2} / 4\right)^{3 / 2}}\right), \quad N_{0}(\tau, y)=\operatorname{Re}\left(\frac{A(\tau+2 a)}{\left(y-(\tau+2 a)^{2} / 4\right)^{3 / 2}}\right)
$$

линейной системы мелкой воды над наклонным дном (см. [24], [25]):

$$
U_{\tau}+N_{y}=0, \quad N_{\tau}+(y U)_{y}=0 .
$$

Решение линейной системы и уравнения Дарбу связаны преобразованием

$$
\begin{array}{cl}
w_{\rho}(u, \rho)=-\frac{u \rho}{2}+\frac{\rho}{2} U\left(-u, \frac{\rho^{2}}{4}\right), & w_{u}(u, \rho)=-\frac{\rho^{2}}{4}-\frac{u^{2}}{2}+N\left(-u, \frac{\rho^{2}}{4}\right), \\
U(\tau, y)=-\tau+\left.\frac{2}{\rho} w_{\rho}(-\tau, \rho)\right|_{\rho=2 \sqrt{y}}, & N(\tau, y)=y+\frac{\tau^{2}}{2}+\left.w_{u}(u, 2 \sqrt{y})\right|_{u=-\tau} .
\end{array}
$$

Соответствующее решение $v(t, y), \eta(t, y)$ нелинейной системы иллюстрирует нелинейный накат волн на наклонный берег (функция дна $D=y$ ) с последующим отражением от берега. При больших амплитудах решение обрушивается (как на рис. 8), а при достаточно малых амплитудах - отражается без обрушения (как на рис. 9). При этом падающая волна в виде "шапочки" отражается в виде так называемой $N$-волны ${ }^{1)}$. В рамках системы (1) это явление изучено с привлечением преобразования Кариера-Гринспана [3] в работах Мазовой и Пелиновского (см., в частности, $[26],[2])$ и применено в задаче о накате на берег волн цунами. Приведенные выше явные формулы алгебраического вида для $U_{0}, N_{0}$ получены в работе [4]. Из графиков, основанных на этих формулах, видно, что нелинейность приводит к укручению профиля и до обрушения волны ее влияние на профиль не слишком существенно. Заметим также, что согласно проведенным исследованиям [26], [2] более $75 \%$ цунами выходят на берег без обрушения.

\footnotetext{
1) В линейном случае после соответствующей нормировки падающая "шапочка" имеет вид "размазанной" $\delta$-функции, а отраженная волна - "размазанной" функции Сохоцкого $1 / z$, и мы имеем так называемую метаморфозу профиля волны после отражения от фокальной (особой) точки $y=0$, связанную со скачком индекса Маслова (см. [4]).
} 


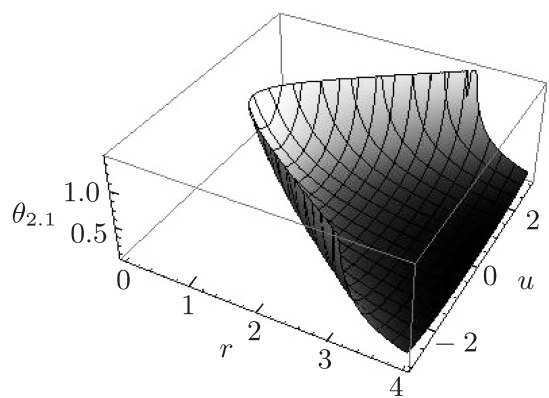

a
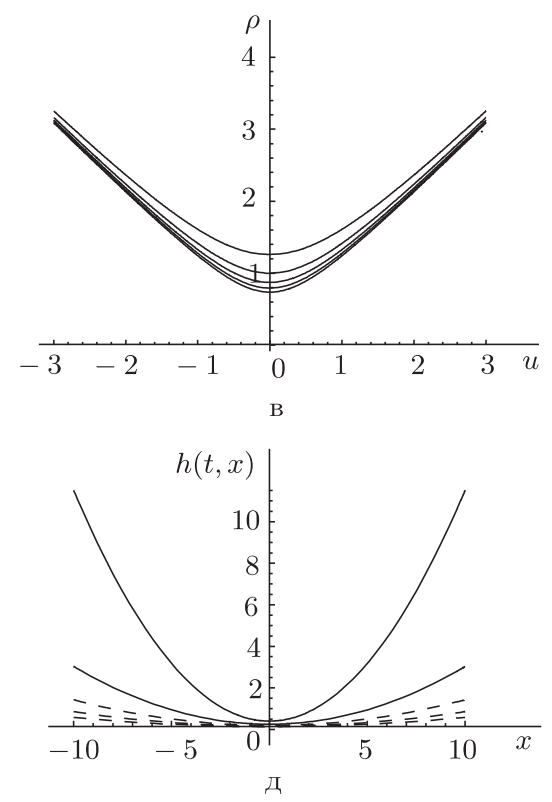

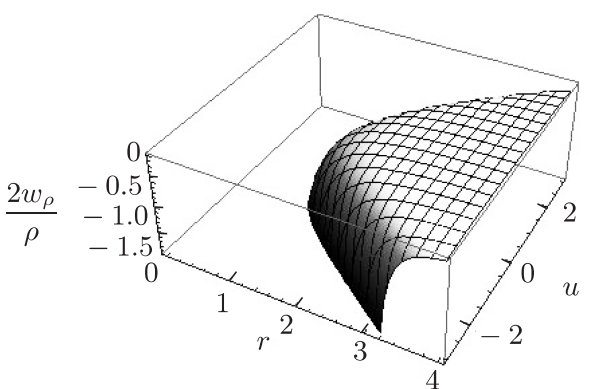

6
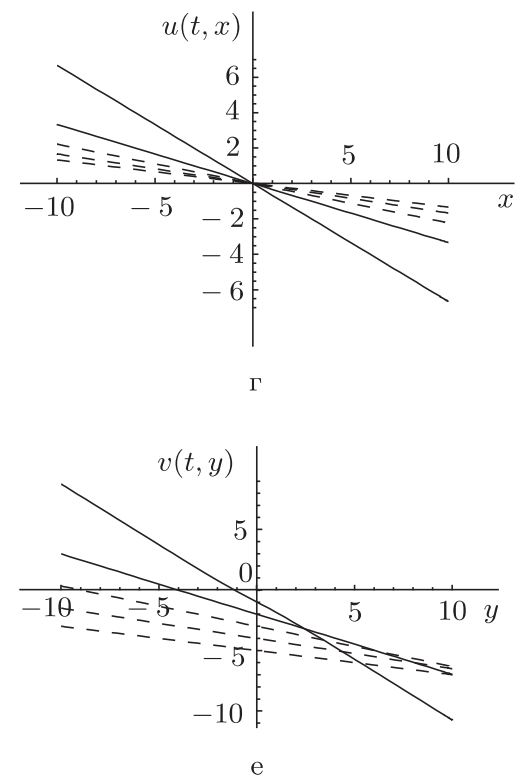

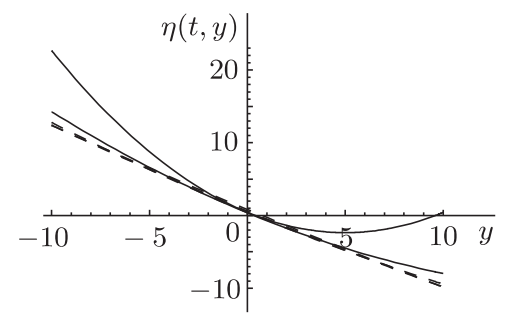

K

Рис. 1. График решения $w=\theta_{2.1}(u, \rho)$ для $c_{0}=1$ (а), график функции $2 w_{\rho} / \rho$ (б), линии уровня функции $2 w_{\rho} / \rho=t$ при $t=-5,-4,-3,-2,-1$ (в) и графики решений нелинейных систем $u(t, x)(г), h(t, x)(д), v(t, y)(\mathrm{e})$, $\eta(t, y)$ (ж) при $t=-5,-4,-3$ (штриховые линии) и $t=-2,-1$ (сплошные линии). 


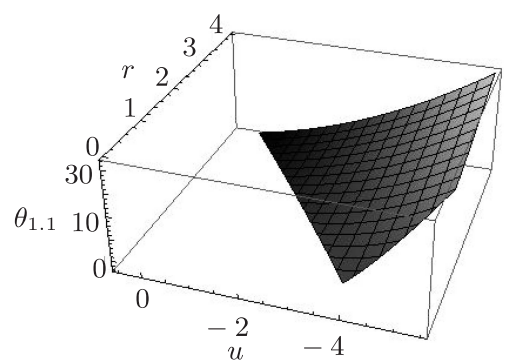

a
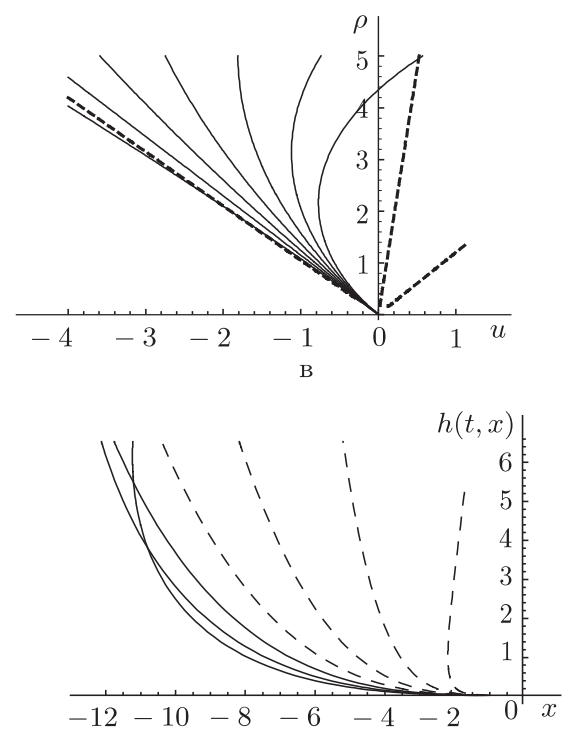

Д

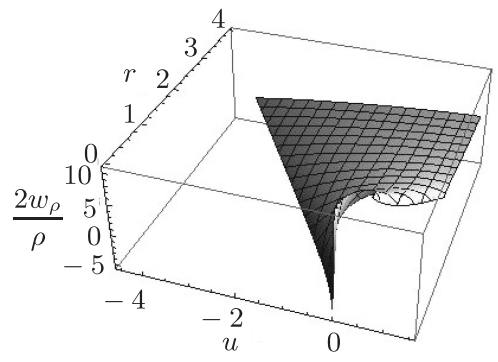

6

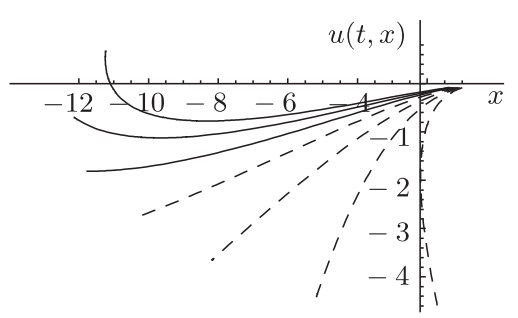

$\Gamma$

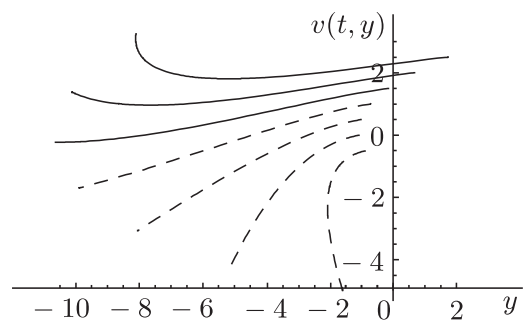

e

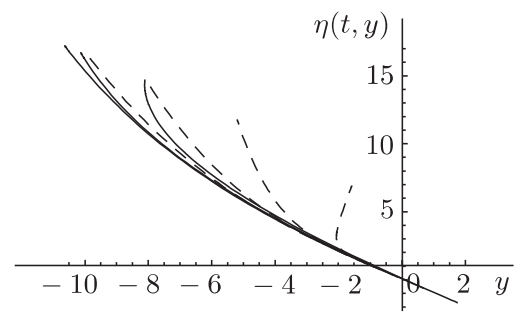

K

Рис. 2. График решения $w=\theta_{1.1}(u, \rho)$ для $\alpha=1.4, c_{1}=-2, c_{2}=1$ (a), график функции $2 w_{\rho} / \rho$ (б), линии уровня функции $2 w_{\rho} / \rho=t$ для $t=$ $-0.5,0,0.5,1,1.5,2,2.5$ (в). На графике $(u, \rho)$ штриховые линии - линии, на которых якобиан $\partial(t, x) / \partial(u, h)$ обращается в нуль. Графики решений $u(t, x)($ г), $h(t, x)($ д), $v(t, y)(\mathrm{e}), \eta(t, y)$ (ж) при $t=-0.5,0,0.5,1$ (штриховые линии) и $r=1.5,2,2.5$ (сплошные линии). На кривых $(u, \rho)$ якобиан обращается в нуль при достаточно больших $r$ и соответственно кривые $u(t, x), h(t, x), v(t, y)$ и $\eta(t, y)$ обрушиваются. 


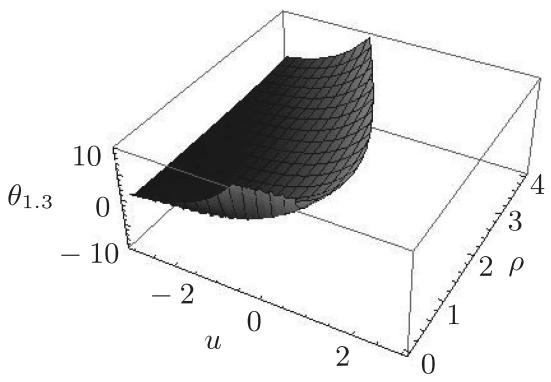

a

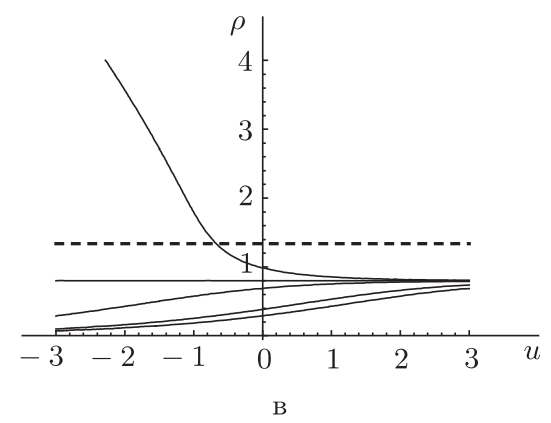

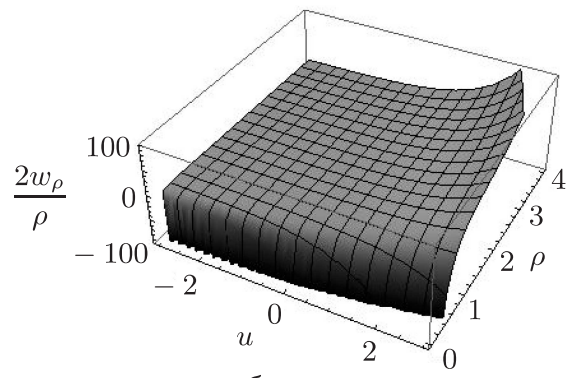

б

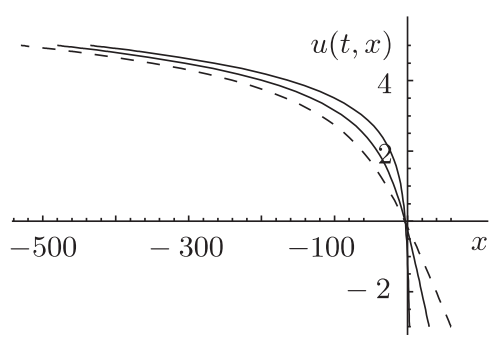

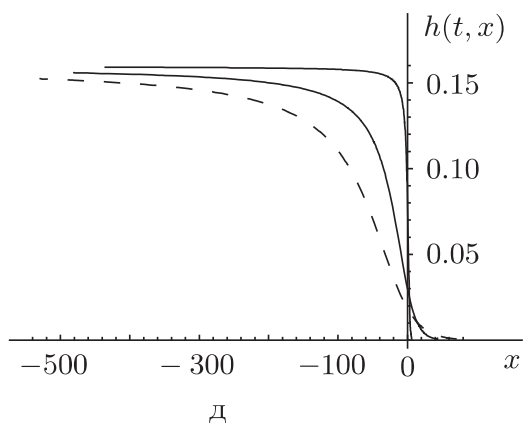

Рис. 3. График решения $w=\theta_{1.3}(u, \rho)$ для $c_{7}=2, c_{8}=1$ (а), график функции $2 w_{\rho} / \rho$ (б), линии уровня функции $2 w_{\rho} / \rho=t$ для $t=-20,-10,-1,0,1$ (в). При $t>0$ линии уровня пересекают линию нулевого якобиана $\partial(t, x) / \partial(u, h)$, (штриховая прямая). Графики решений $u(t, x)($ г) и $h(t, x)$ (д) при $t=-20$ (штриховые линии) и $t=-10,-1$ (сплошные линии).

Преобразование (27) действует локально: решение $w(u, \rho)$ в области $(u, \rho)$, где не обращается в нуль якобиан $\partial(t, x) / \partial(u, h)$, определяет решение $u(t, x), h(t, x)$ в некоторой области $(t, x)$. Приведем несколько примеров.

Пример 1. Решение $u=2 x / 3 t, h=x^{2} / 9 t^{2}+\left(2 c_{0}\right)^{2 / 3} / 4 t^{2 / 3}$, соответствующее $\theta_{2.1}$ для $c_{0}>0$ (см. рис. 1$)$, решение $u, h$, соответствующее $\theta_{1.3}$ для $c_{7}=2, c_{8}=1$ (см. рис. 4), и решение $u=x / t, h=c_{6} / 2 t, c_{6}<0$, соответствующее $\theta_{1.2}$, определены при всех $x$ при $t<0$. Решение, соответствующее $\theta_{1.5}$ для $c_{11}=0, c_{12}=1$, определено при $x<0$ при $t<t_{\mathrm{cr}} \approx-1$ (см. рис. 6). Решение, соответствующее $\operatorname{Re} \theta_{1.6}$ для $\beta=0$, $c_{17}=-1, c_{18}=0$, определено при всех $x$ при $t>t_{\mathrm{cr}} \approx 0.6$ (см. рис. 7 ). Перечисленные решения существуют на интервалах по $x$, которые не зависят от $t$. 


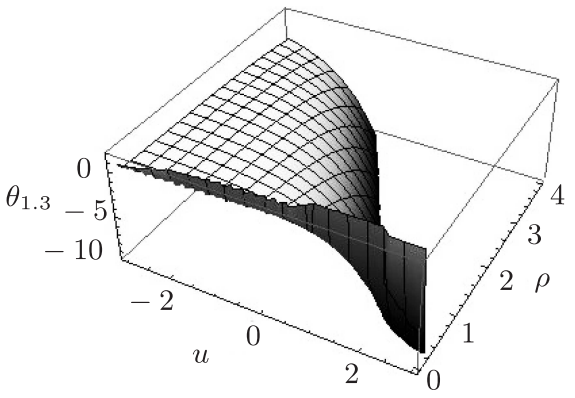

a

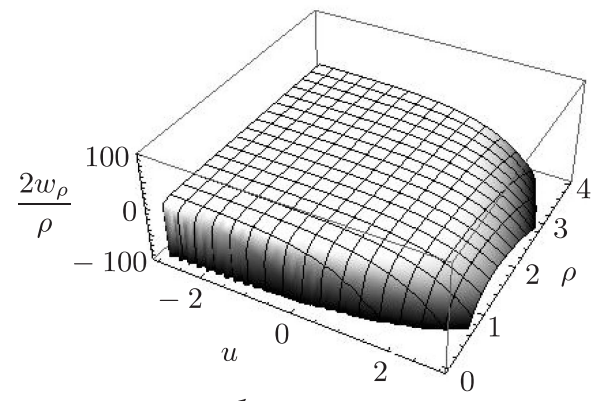

б

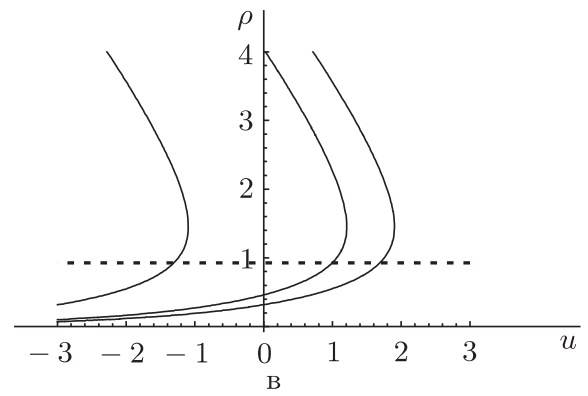

Рис. 4. График решения $w=\theta_{1.3}(u, \rho)$ для $c_{7}=-2, c_{8}=1$ (а), график функции $2 w_{\rho} / \rho$ (б) и линии уровня $2 w_{\rho} / \rho=t$ для $t=-20,-10,-1$ (в).

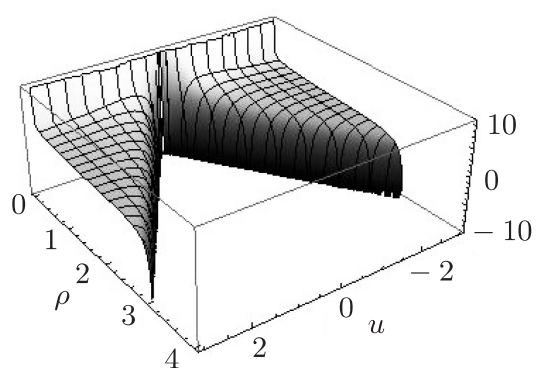

a
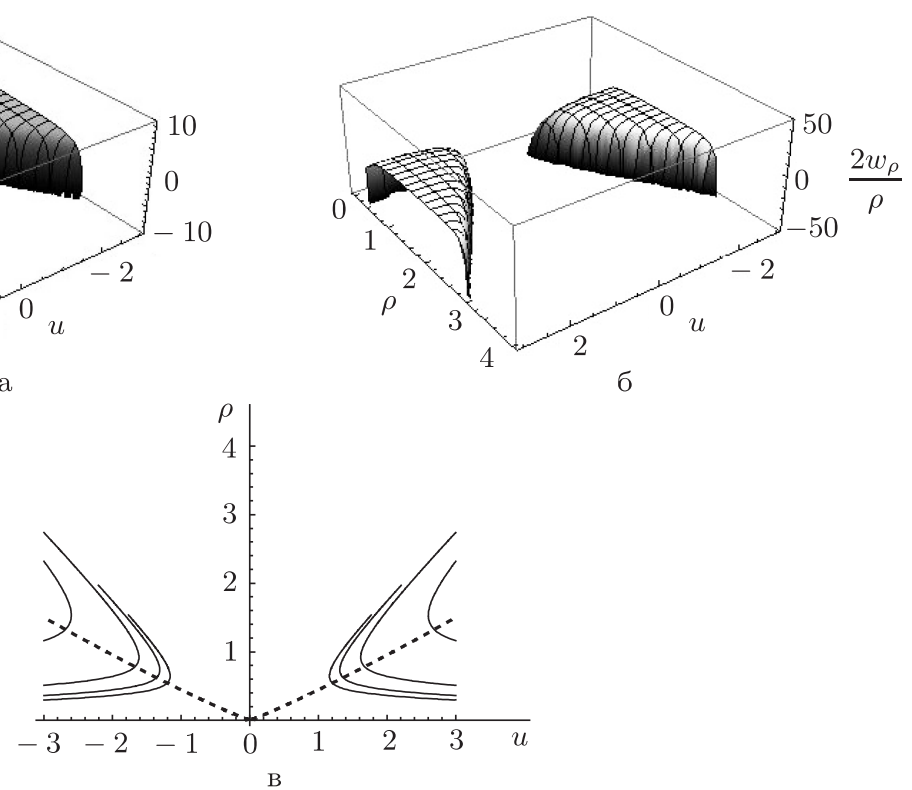

Рис. 5. График решения $w=\theta_{1.4}(u, \rho)$ для $c_{9}=1, c_{10}=2(\mathrm{a})$, график функции $2 w_{\rho} / \rho$ (б), линии уровня функции $2 w_{\rho} / \rho=t$ для $t=-15,-10,-5,-1$ (в). Если $c_{10} \neq 0$, то кривые $(u, \rho)$ пересекают линию нулевого якобиана и "глобального" решения для волн на воде не существует. 


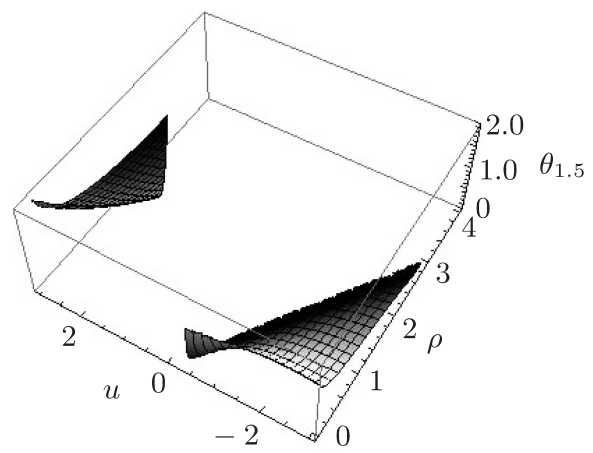

a

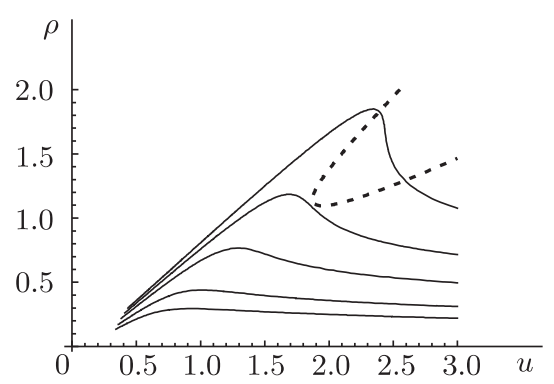

B

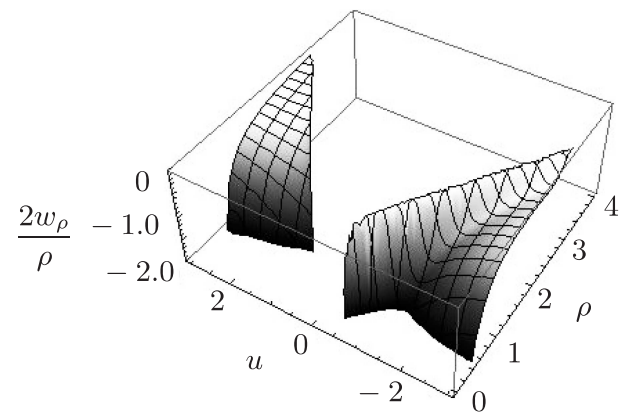

б

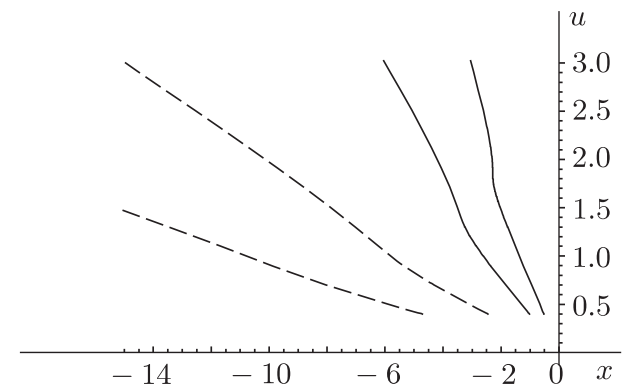

$\Gamma$

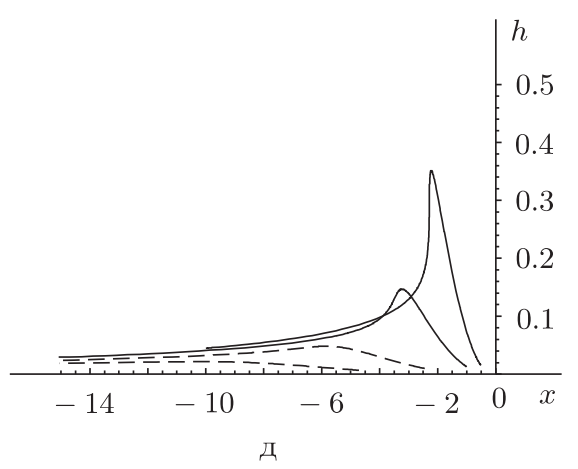

Рис. 6. График решения $w=\theta_{1.5}(u, \rho)$ для $c_{11}=0, c_{12}=1$ (a), график функции $w_{\rho} / \rho(б)$, линии уровня функции $2 w_{\rho} / \rho=t$ для $t=$ $-10,-5,-2,-1,-0.5$ (в). При $t>t_{\mathrm{cr}} \approx-1$ эти кривые пересекают линию нулевого якобиана (штриховая линия). Графики решений $u(t, x)(г)$ и $h(t, x)($ д) для $t=-10,-5$ (штриховые линии) и $t=-2,-1$ (сплошные линии).

ПримеР 2. Решения уравнения Дарбу могут определять решения нелинейной системы (1) на промежутке по $x$, меняющемся со временем (граница определяется из условия $h(t, x)=0)$. Так, $\theta_{1.4}$ при $c_{10}=0$ определяет решение

$$
u=\frac{2 x}{3 t}, \quad h=\frac{x^{2}}{9 t^{2}}-\frac{\left(2 c_{9}\right)^{2 / 3}}{4 t^{2 / 3}}
$$




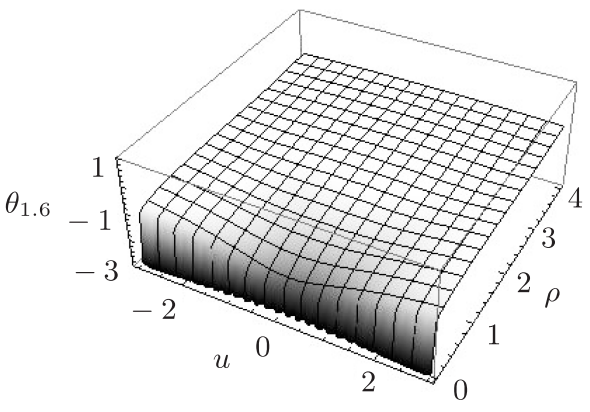

a

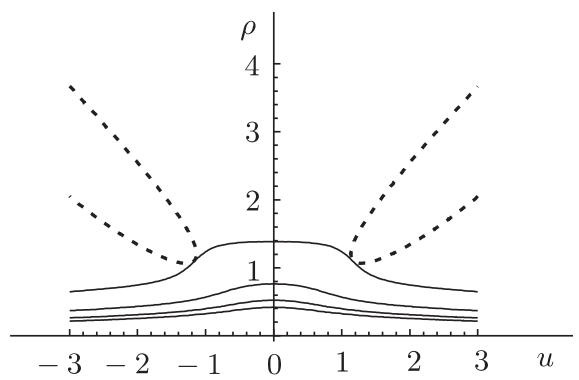

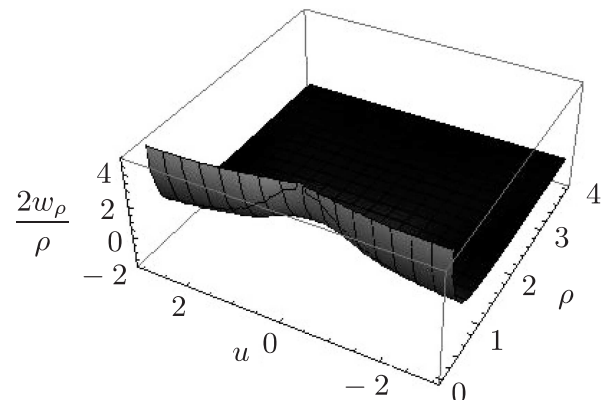

б

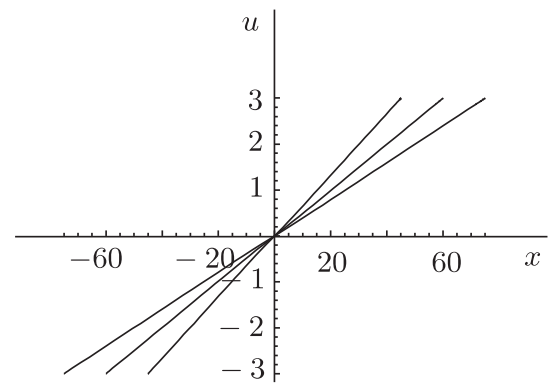

$\Gamma$

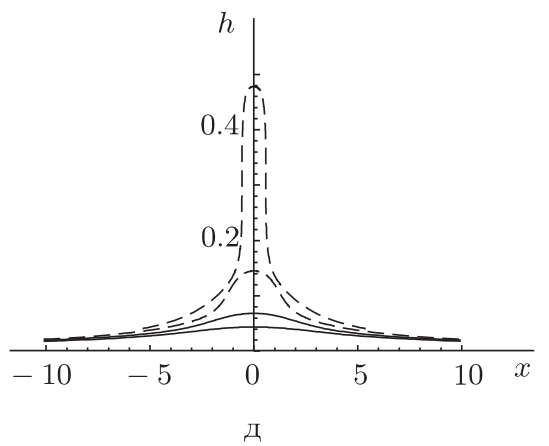

Рис. 7. График решения $w=\operatorname{Re}\left(\theta_{1.6}(u, \rho)\right)$ для $\beta=0, c_{17}=-1$, $c_{18}=0\left(\right.$ a), график функции $w_{\rho} / \rho(б)$, линии уровня функции $2 w_{\rho} / \rho=t$ для $t=0.6,2,4,6$ и линии нулевого якобиана (штриховые линии) (в). Графики решений $u(t, x)(г)$ и $h(t, x)$ (д) для $t=0.6,2$ (штриховые линии) и $t=4,6$ (сплошные линии).

при $t>0$ на промежутках $|x| \geqslant x_{0}(t) \equiv(3 / 2) \sqrt[3]{2 c_{9}} t^{2 / 3}$ с переменным пределом $\pm x_{0}(t)$. Решение $U_{0}, N_{0}$ линейной системы мелкой воды с наклонным дном определяет решения нелинейной системы на интервале с переменным левым пределом (см. рис. 8, $9)$, которые могут быть определены как при всех $t$, если амплитуда $A$ достаточно маленькая, так и при $t \in\left(-\infty, t_{\mathrm{cr}_{1}}\right) \cup\left(t_{\mathrm{cr}_{2}}, \infty\right)$ для больших амплитуд. 


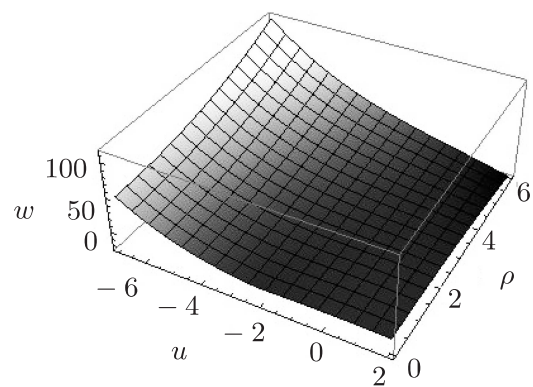

a

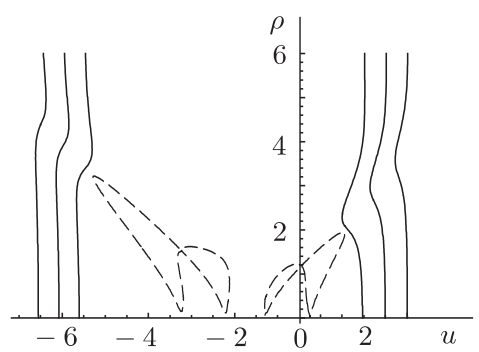

B

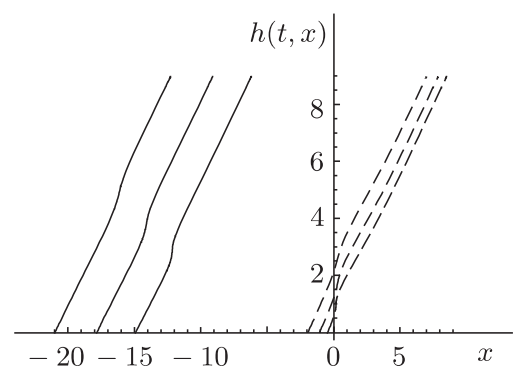

Д

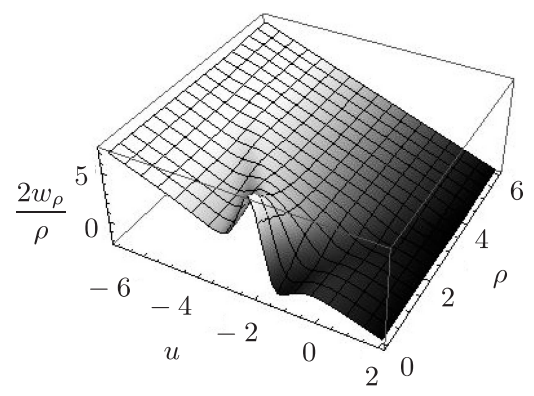

б
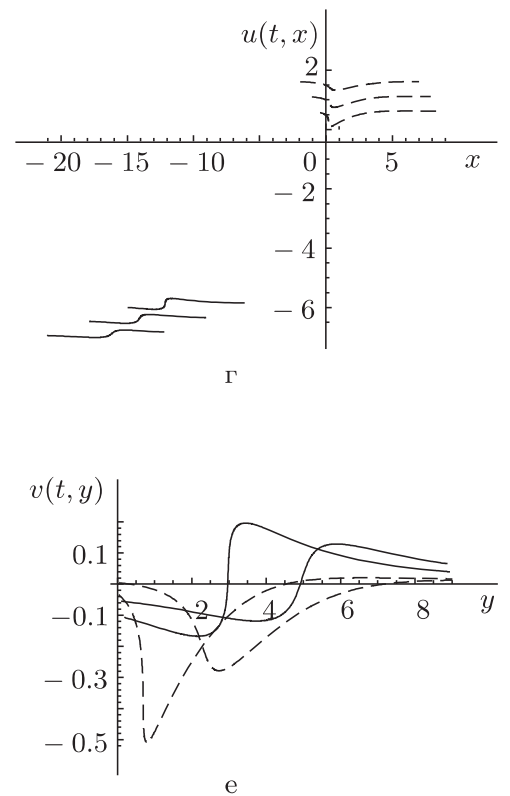

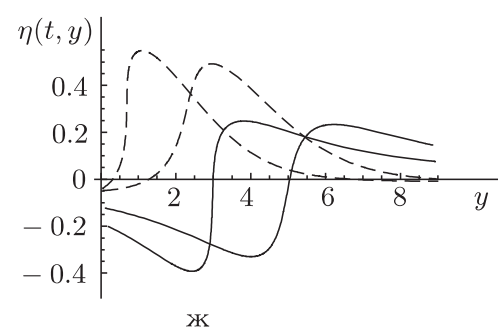

Рис. 8. Графики решения $w(u, \rho)$ (а) и функции $2 w_{\rho} / \rho(б)$, соответствующие решению $U_{0}, N_{0}$ линейной системы для $A=(1+i) / 4,2 a=-2-i$, линии уровня функции $2 w_{\rho} / \rho=t$ для $t=-2,-1.5,-1$ и $t=5.5,6,6.5$ (в), при $t \in\left(t_{\mathrm{cr}_{1}}, t_{\mathrm{cr}_{2}}\right) \approx(-0.9,5.3)$ линии уровня пересекают линии нулевого якобиана (штриховые линии). Графики решений $u(t, x)(г), h(t, x)$ (д) при $t=$ $-2,-1.5,-1,5.5,6,6.5$ и графики $v(t, y)(\mathrm{e}), \eta(t, y)$ (ж) при $t=-2,-1,5.5,6.5$; при $t<t_{\mathrm{cr}_{1}}-$ штриховые линии, при $t>t_{\mathrm{cr}_{2}}-$ сплошные. 


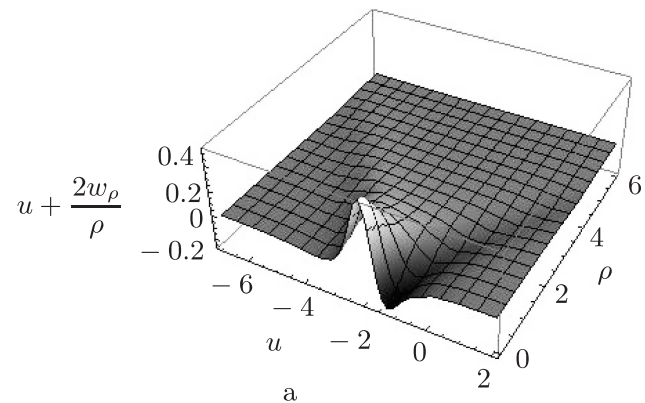

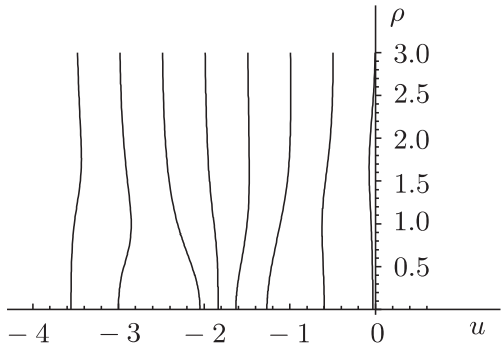

6

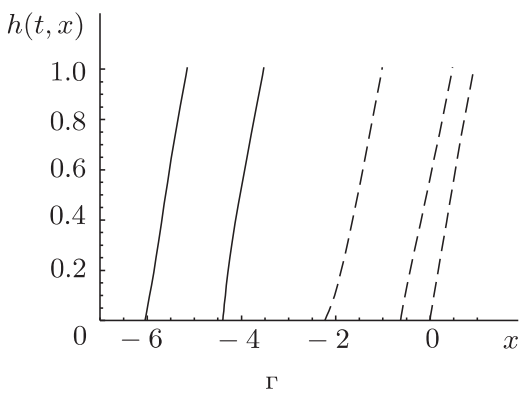

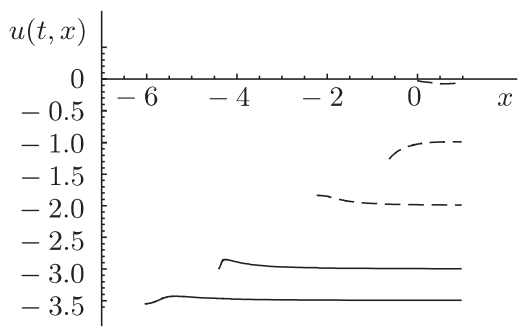

$\mathrm{B}$

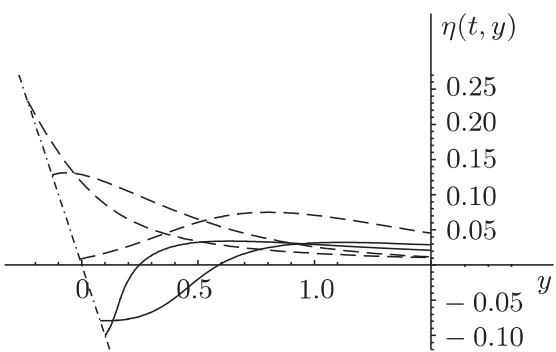

д

Рис. 9. Графики $u+2 w_{\rho} / \rho$ (а) и линии уровня функции $2 w_{\rho} / \rho=t$ для $t=$ $0,0.5,1,1.5,2,2.5,3,3.5$ (б), где решение $w(u, \rho)$ соответствует решению $U_{0}, N_{0}$ линейной системы с малой амплитудой: $A=(1+i) / 40,2 a=-2-i$. Графики $u(t, x)($ в), $h(t, x)($ г) и $\eta(t, y)$ (д) при $t=0,1,2$ (штриховые линии) и $t=3,3.5$ (сплошные линии). Штрихпунктирная линия на графике $\eta(t, y)$ обозначает уровень дна (глубина $D=y)$. Якобиан $\partial(t, x) / \partial(u, h)($ и $\partial(t, y) / \partial(v, \eta))$ нигде не обращается в нуль, так что решение нелинейной системы существует при всех $t$.

Пример 3. В случае, когда на кривых $(u, \rho)$, определяемых уравнением $2 w_{\rho} / \rho=$ $t=$ const, якобиан $\partial(t, x) / \partial(u, h)$ обращается в нуль, решения уравнения Дарбу могут определять решения нелинейной системы только локально, так как при некотором $x$ последние обрушиваются и далее формулы (27) определяют многозначные функции $u(t, x), h(t, x)$. Такая ситуация имеет место, например, для решения $\theta_{1.1}$ при $\alpha=1.4, c_{1}=-2, c_{2}=1$ (см. рис. 2 ), для решения $\theta_{1.3}$ при $c_{7}=2, c_{8}=1$ при $t>0$ 
и $c_{7}=-2, c_{8}=1$ (см. рис. 3,4$)$, для решения $\theta_{1.4}$ при $c_{9}=1, c_{10}=2$ (см. рис. 5), для решения $\theta_{1.5}$ при $c_{11}=0, c_{12}=1$ при $t>t_{\mathrm{cr}} \approx-1$, для решений, соответствующих решениям $N_{0}, U_{0}$ линейной системы мелкой воды при $t \in\left(t_{\mathrm{cr}_{1}}, t_{\mathrm{cr}_{2}}\right)$.

ЗАмЕчАниЕ 1. Если известно семейство решений $w(x, t, \rho)$ линейной гиперболической системы, зависящих от параметра (параметров) $\rho \in \Omega$, то часто можно построить новые решения, умножив это семейство на некоторую функцию (даже матричнозначную функцию) $g(\rho)$, зависящую от параметра (параметров) $\rho \in \Omega$, и проинтегрировав результат по этому параметру (этим параметрам):

$$
w_{\text {new }}(u, \rho)=\int_{\Omega} w(x, t, \rho) g(\rho) d \rho .
$$

\section{6. ВЫРОЖДЕННОЕ РЕШЕНИЕ}

Система (1) имеет тривиальное вырожденное решение: $u=$ const, $h=$ const. Отличное от него нетривиальное вырожденное решение системы (1) удовлетворяет условию равенства нулю якобиана $\partial(u, h) / \partial(t, x)=0$, из которого следует, что $h=$ $\Phi(u)$, т. е. нетривиальное вырожденное решение системы (1) является ее простой волной [9].

Для системы (1) поиск этого вырожденного решения не вызывает никаких затруднений. Подстановка в систему (1) и условие нетривиальности решения дают уравнение простой волны: $\left(\Phi^{\prime}\right)^{2}=\Phi$. Интегрирование этого уравнения приводит к двум выражениям для величины $h$, подстановка которых в систему (1) дает соответствующие уравнения для определения $u$ - параметра простой волны. Последние уравнения оказываются уравнениями Хопфа специальных видов, интегрирование которых определяет неявно параметр $u$. В результате множество нетривиальных вырожденных решений системы (1) исчерпывается следующими решениями:

1) $h=0$, а функция $u$ определяется неявно уравнением

$$
f(u, x-t u)=0 ;
$$

2) $h=u / 2+c$, а функция $u$ определяется неявно уравнением

$$
f\left(u, x-t\left(\frac{3 u}{2}+c\right)\right)=0,
$$

где $c$ - произвольная вещественная постоянная, а $f$ - произвольная непостоянная функция.

Система (2) имеет тривиальное вырожденное решение: $v=0, \eta=$ const. Отличное от него нетривиальное вырожденное решение системы (2) удовлетворяет условию равенства нулю якобиана $\partial(v, \eta) / \partial(\tau, y)=0$, из которого следует, что $\eta=F(v)$, т. е. нетривиальное вырожденное решение системы (2) является ее простой волной.

Система (2) для этой простой волны принимает вид

$$
v_{\tau}+\left(v+F^{\prime}\right) v_{y}=0, \quad\left(\left(F^{\prime}\right)^{2}-F-y\right) v_{y}=v .
$$

Условием совместности этой системы является равенство

$$
v\left(2 v+F^{\prime}+v F^{\prime \prime}\right)=0
$$


из которого следует, что функция $F$ имеет вид

$$
F=c^{1} \ln v-\frac{v^{2}}{2}+c^{2}
$$

где $c^{1}, c^{2}$ - произвольные вещественные постоянные.

Подстановка функции (52) в систему (51) и интегрирование полученных уравнений дает уравнение, которое определяет неявно параметр простой волны $v$ :

$$
c^{1}\left(v \ln v+v+\frac{1}{v}-\tau\right)+c^{2} v-\frac{v^{3}}{2}+y v=c^{3},
$$

где $c^{1}, c^{2}, c^{3}$ - произвольные вещественные постоянные.

Таким образом, множество нетривиальных вырожденных решений системы (2) исчерпывается следующими решениями:

$$
\eta=c^{1} \ln v-\frac{v^{2}}{2}+c^{2}
$$

а функция $v$ определяется неявно уравнением (53).

\section{7. ИСТОРИЧЕСКИЕ КОММЕНТАРИИ}

Системы уравнений мелкой воды (1) и (2) достаточно давно изучаются и используются для описания течений в руслах рек и цунами [1]-[3]. Система линейных уравнений (11), а точнее, уравнение второго порядка, которое может быть получено из этой системы, имеет давнюю историю.

В 1738 году Бернулли получил уравнение для описания малых колебаний тяжелой нити, подвешенной за один конец в поле силы тяжести:

$$
\frac{\partial^{2} u}{\partial t^{2}}=\frac{\partial}{\partial x}\left(x \frac{\partial u}{\partial x}\right)
$$

Позднее, в 1781 году, оно было получено также Эйлером. Это уравнение можно считать родоначальником при исследовании функций Бесселя, как это описано в монографии [27] (современное изложение можно найти в книге [28]).

В области гиперболичности этого уравнения $x>0$ можно сделать замену переменной $x=r^{2} / 4$, что дает уравнение

$$
\frac{\partial^{2} u}{\partial t^{2}}=\frac{\partial^{2} u}{\partial r^{2}}+\frac{1}{r} \frac{\partial u}{\partial r}
$$

Уравнение (55) с точностью до обозначений совпадает с уравнением (28). Бернулли и Эйлер искали решение этого уравнения методом разделения переменных. Представление общего решения в интегральном виде было дано Пуассоном в 1823 году [29]:

$$
u(r, t)=\int_{0}^{\pi} f(r \cos \omega+t) d \omega+\int_{0}^{\pi} F(r \cos \omega+t) \ln \left(r \sin ^{2} \omega\right) d \omega,
$$

где $f$ и $F$ - произвольные функции. Однако ни в одной из известных авторам последующих работ это интегральное решение уравнения (55) не упоминалось. 
Отметим также, что уравнение (55) является радиальной частью двумерного волнового уравнения в полярных координатах, которое изучалось Эйлером в 1764 году методом разделения переменных. Методами группового анализа уравнение (55) также исследовалось в [13], [30].

Уравнение (55) является частным случаем уравнения Эйлера-Пуассона-Дарбу, которое записывается в канонической форме [10], [11]

$$
\frac{\partial^{2} u}{\partial x \partial y}-\frac{b^{\prime}}{x-y} \frac{\partial u}{\partial x}+\frac{b}{x-y} \frac{\partial u}{\partial y}=0 .
$$

Кроме теории колебаний струн и мембран, этот класс уравнений возник также в работе Римана о распространении волн конечной амплитуды. Современное изложение дано в [31]. В самое последнее время были исследованы симметрийные свойства уравнения Эйлера-Пуассона-Дарбу для политропного газа [21]. Однако уравнение (55) не попадает в множество уравнений для политропного газа.

\section{8. ЗАКЛЮЧЕНИЕ}

Для систем уравнений одномерных моделей мелкой воды, описывающих распространение поверхностных волн над ровным и наклонным дном, получены формулы общего вида их невырожденных решений, которые выражаются через решения уравнения Дарбу. Найдены существенно различные (не связанные обратимыми точечными преобразованиями) инвариантные решения этого уравнения и соответствующие им решения исходных систем. Также построены и все вырожденные решения исходных систем. В результате создана база данных бесконечного множества точных решений систем уравнений одномерной модели мелкой воды с ровным и наклонным дном. С помощью графических построений выяснен физический смысл некоторых полученных решений, выраженных через специальные функции. Приведены некоторые исторические сведения, касающиеся исследования линейных уравнений, к которым сводятся нелинейные уравнения одномерной мелкой воды.

Благодарности. Авторы благодарят О. В. Капцова за полезные обсуждения решений уравнения Эйлера-Пуассона-Дарбу. Работа выполнена при финансовой поддержке РФФИ (проекты № 11-01-12075-офи_м, 12-01-00648, 11-01-00973_а), Программы поддержки ведущих научных школ (проект НШ-706.2012.1), грантов Правительства РФ для государственной поддержки научных исследований, проводимых под руководством ведущих ученых (договор № 1.G34.31.0054) и для государственной поддержки научных исследований коллективами под руководством приглашенных исследователей (соглашение № 8504), а также Министерства образования и науки РФ (государственный контракт № 2014/138, проект № 435).

\section{Список литературы}

[1] Дж. Дж. Стокер, Волны на воде. Математическая теория и приложения, ИЛ, М., 1959.

[2] Е. Н. Пелиновский, Гидродинамика волн иунами, ИПФ РАН, Нижний Новгород, 1996.

[3] G. F. Carrier, H. P. Greenspan, J. Fluid Mech., 4:1 (1958), 97-109.

[4] С. Ю. Доброхотов, Б. Тироцци, УМН, 65:1(391) (2010), 185-186.

[5] I. Didenkulova, E. Pelinovsky, Nonlinearity, 24:3 (2011), R1-R18. 
[6] S. Yu. Dobrokhotov, V.E. Nazaikinskii, B. Tirozzi, Russ. J. Math. Phys., 17:4 (2010), 434-447.

[7] Д. С. Миненков, Матем. заметки, 92:5 (2012), 721-730.

[8] Дж. Уизем, Линейные и нелинейные волны, Мир, М., 1977.

[9] Ю. А. Чиркунов, С. В. Хабиров, Элементы симметрийного анализа дифференциальных уравнений механики сплошной среды, НГТУ, Новосибирск, 2012.

[10] Л. В. Овсянников, Групповой анализ дифференииалъных уравнений, Наука, М., 1978.

[11] G. Darboux, Leçons sur la théorie générale des surfaces et les applications géométriques du calcul infinitésimal, v. II, Gauthier-Villars, Paris, 1915.

[12] Л. В. Овсянников, Журн. прикл. мех. и техн. физ., 1960, № 3, 126-145.

[13] W. Miller, Jr., SIAM J. Math. Anal., 4:2 (1973), 314-328.

[14] О.В. Капцов, Методы интегрирования уравнений с частными производными, Физматлит, М., 2009.

[15] Ю. А. Чиркунов, Докл. АН СССР, 314:1 (1990), 155-159.

[16] Ю. А. Чиркунов, Сиб. журн. индустр. матем., 13:1 (2010), 140-149.

[17] Ю. А. Чиркунов, Сиб. журн. индустр. матем., 13:4 (2010), 131-140.

[18] Ю. А. Чиркунов, Матем. заметки, 87:1 (2010), 122-129.

[19] Ю.А. Чиркунов, Групповой анализ линейных и квазилинейных дифференциальных уравнений, НГУЭУ, Новосибирск, 2007.

[20] Ю. А. Чиркунов, Докл. РАН, 426:5 (2009), 605-607.

[21] В. А. Аксенов, Докл. РАН, 381:2 (2001), 176-179.

[22] Э. Т. Уиттекер, Дж. Н. Ватсон, Курс современного анализа, Ч. 2, Наука, М., 1963.

[23] И.С. Градштейн, И.М. Рыжик, Таблицы интегралов, сумм, рядов и произведений, Физматлит, М., 1971.

[24] С. Ю. Доброхотов, С. Я. Секерж-Зенькович, Матем. заметки, 88:6 (2010), 942-945.

[25] С. Ю. Доброхотов, С.Б. Медведев, Д. С. Миненков, Матем. заметки, 93:5 (2013), $716-727$.

[26] Р. Х. Мазова, Е. Н. Пелиновский, "Накат волн цунами на берег без обрушения", Волнъь и дифракиия, т. 2, ИРЭ АН СССР, М., 1981, 265-268.

[27] Г.Н. Ватсон, Теория бесселевых функиий, ИЛ, М., 1949.

[28] Н.С. Кошляков, Основные дифференииальные уравнения математической физики, Физматгиз, М., 1962.

[29] M. Poisson, École Polytechnique, 12, 19 (1823), 215-248.

[30] У. Миллер, Симметрия и разделение переменных, Мир, М., 1981.

[31] Р. Мизес, Математическая теория течений сжимаемой жидкости, ИЛ, М., 1961. 